\title{
CINÉTICA DE CRISTALIZAÇÃO DE SACAROSE EM CRISTALIZADOR DE LEITO VIBRADO
}

\author{
D. B. QUINTINO ${ }^{1}$, R. A. MALAGONI ${ }^{*}$ \\ ${ }^{1}$ Universidade Federal de Uberlândia, Faculdade de Engenharia Química \\ *e-mail: malagoni@feq.ufu.br
}

\begin{abstract}
RESUMO
A cristalização é a principal operação unitária no processo de fabricação da sacarose comercial, este processo pode ocorrer naturalmente ou em equipamentos denominados cristalizadores. Este estudo teve como objetivo determinar a equação de cinética de crescimento de cristais de sacarose em um cristalizador batelada de leito vibrado. A fim de definir qual a melhor condição operacional para simultaneamente obter maior produtividade e tamanho médio de cristais, seguiu-se um planejamento de experimentos no qual as variáveis supersaturação, vibração do meio e tempo de cristalização foram observadas. Após a obtenção da condição de resposta máxima para produtividade e diâmetro do cristal, acompanhou-se a variação do crescimento cristalino em seis diferentes tempos, através da análise granulométrica dos cristais. Observou-se a alta influência da supersaturação aliada à vibração do meio e o rápido crescimento no início do processo, com decaimento a partir do décimo quinto minuto de operação, possibilitando a obtenção de cristais com granulometria semelhante aos comercialmente disponíveis.
\end{abstract}

\section{INTRODUÇÃO}

\subsection{A Sacarose}

Também conhecida como açúcar comercial, a sacarose é amplamente estudada em pesquisas voltadas para alimentos, pois desempenha um importante papel como ingrediente e conservante em várias formulações e processos tecnológicos (QUINTAS et al., 2005).

Segundo dados da FAO (2014) (Food and Agriculture Organization of United Nations), a produção mundial de açúcar na safra de 2012/2013 foi de 182,4 milhões de toneladas. O Brasil, maior produtor mundial destaca-se como $22 \%$ da produção global. Em nosso país, a cana-de-açúcar é cultivada em diversas regiões, com diferentes períodos de colheita nas regiões Nordeste e Sudeste, desta última, destaca-se o Estado de São Paulo, responsável por mais da metade da produção nacional (ANDRADE et al., 2010).

\subsection{Cristalização em Leito Vibrado}

A cristalização é um processo de separação sólido-líquido no qual as moléculas são transferidas de um soluto dissolvido na fase líquida para uma fase sólida (MULLIN, 2001; JONES, 2002). Esta operação unitária é considerada como um processo de baixo custo operacional na produção de sólidos a partir de soluções impuras (NAGY et al., 2013; CAMILO et al., 2010; MYERSON., 2002). $\mathrm{Na}$ produção de cristais de sacarose, a cristalização é a etapa principal de produção, sendo governada por dois processos principais: nucleação e crescimento cristalino.

Cristalizadores batelada têm sido amplamente utilizados em estudos de cristalização devido ao seu simples modo de 
operação e controle de variáveis, sua fácil construção, possuírem pequenos custos de manutenção e possibilitar diversas quantidades de batelada (OUIAZZANE et al., 2007).

No processo de cristalização em leito vibrado, a condição ótima de operação, é aquela na qual a condição de produtividade é maximizada enquanto os cristais possuem tamanho adequado, significando que, para este processo, ao obter-se a condição operacional de máxima produtividade, a análise cinética de crescimento cristalino, torna-se imprescindível para manutenção da produção dentro da especificação e com aparência agradável ao consumidor (CHENG \& CHOU, 1993; ULRICH \& JONES, 2004; TEIXEIRA et al., 2012).

O objetivo deste estudo foi obter uma configuração operacional de alta produtividade de cristais, e obter a cinética de cristalização dos mesmos através do acompanhamento da variação da dimensão os cristais durante o processo de cristalização.

\section{MATERIAL E MÉTODOS}

Os experimentos de cristalização de sacarose foram realizados no Laboratório de Cristalização da Faculdade de Engenharia Química (FEQUI) da Universidade Federal de Uberlândia (UFU), os compostos químicos utilizados são relacionados na Tabela 1 .

Tabela 1 - Compostos químicos utilizados nos ensaios de cristalização de sacarose.

\begin{tabular}{cc}
\hline Reagente & Fabricante \\
\hline Açúcar Cristal & Caeté LTDA \\
Água deionizada & Gehaka CG-200 \\
Etanol & Cinética LTDA, \\
& $(99,5 \%$ - pureza $)$ \\
\hline
\end{tabular}

Os equipamentos utilizados nos ensaios de cristalização são relacionados na Tabela 2, nesta, é observado tanto o modelo quanto à marca destes.
Tabela 2 - Equipamentos utilizados nos experimentos de cristalização em leito vibrado.

\begin{tabular}{cc}
\hline Equipamento & Marca e Modelo \\
\hline Cristalizador & Leito vibrado \\
Banho termostatizado & Tecnal, TE-184 \\
Motor elétrico & WEG, 0,75CV \\
Inversor de frequência & WEG, CFW-08 \\
Estufa & Medicate, modelo \\
Agitador de peneiras & MD 1.3 \\
\hline
\end{tabular}

\subsection{Cristalizador de Leito Vibrado}

O cristalizador de leito vibrado foi confeccionado em aço inoxidável, encamisado internamente, possui formato tronco-cônico e sua seção cônica tem angulação de $65^{\circ}$ com a horizontal, seu diâmetro superior e inferior possuem respectivamente $0,155 \mathrm{~m}$ e $0,064 \mathrm{~m}$. Os equipamentos responsáveis pela transmissão da energia mecânica ao leito são visualizados na Figura 1, os quais são: eixo vibratório (A) e excêntrico (B).

Figura 1 - Equipamentos que transferem a energia mecânica ao leito: eixo vibratório (A) e excêntrico (B).

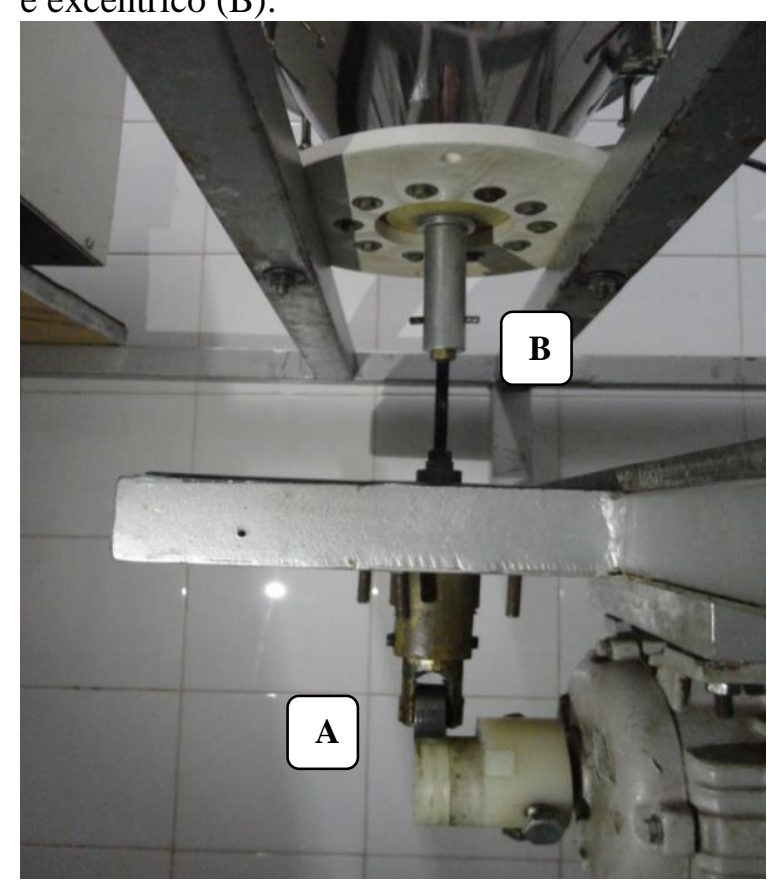

Fonte: Quintino, 2014. 
A energia transmitida ao leito foi gerada pelo motor de corrente alternada, este teve sua velocidade controlada através do inverso de frequência. O sistema de agitação da solução do cristalizador é realizado por meio de pratos perfurados fixados ao eixo central de equipamento. Este tem sua constituição formada por dois discos perfurados confeccionados em aço inoxidável, uma presilha e três anéis de alumínio. Os discos de maior e menor dimensão possuem respectivamente $0,08 \mathrm{~m}$ e $0,06 \mathrm{~m}$ de diâmetro, são também respectivamente perfurados por 40 e 16 furos circulares de $5,0 \times 10^{-3} \mathrm{~m}$.

Na Figura 2 é visualizado o interior do cristalizador tronco-cônico com o sistema de vibração montado. Demais dimensões e especificações do cristalizador podem ser encontradas em Quintino (2014) e Malagoni (2010).

Figura 2 - Sistema de vibração da solução no cristalizador batelada.

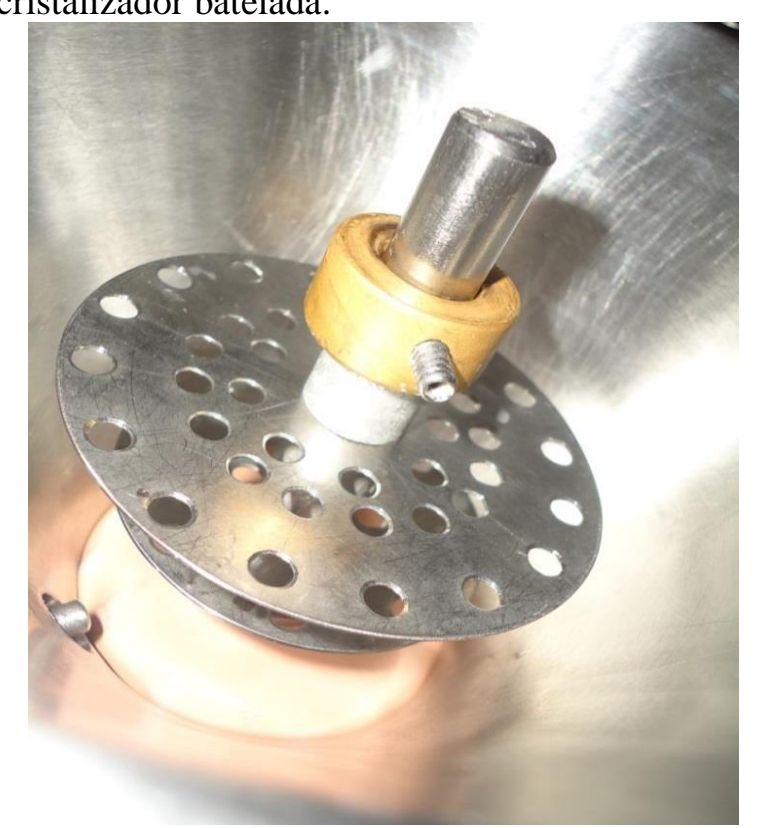

Fonte: Quintino, 2014.

\subsection{Planejamento de Experimentos}

Os experimentos obedeceram a um Planejamento Composto Central (PCC) constituído de 17 experimentos. Os ensaios objetivaram determinar a produção máxima de cristais juntamente com o maior tamanho obtido, analisaram-se duas respostas operacionais, produtividade e diâmetro médio de Sauter, através da variação da supersaturação $(S)$, intensidade de vibração $(\Gamma)$ e tempo $(t)$, conforme Tabela 3.

Tabela 3 - Planejamento Composto Central (PCC).

\begin{tabular}{cccc}
\hline Ensaios & $S$ & $\Gamma$ & $t(\min )$ \\
\hline 1 & 1,05 & 0,433 & 41,75 \\
2 & 1,17 & 0,433 & 41,75 \\
3 & 1,05 & 1,093 & 41,75 \\
4 & 1,17 & 1,093 & 41,75 \\
5 & 1,05 & 0,433 & 108,5 \\
6 & 1,17 & 0,433 & 108,5 \\
7 & 1,05 & 1,093 & 108,5 \\
8 & 1,17 & 1,093 & 108,5 \\
9 & 1,02 & 0,763 & 75 \\
10 & 1,20 & 0,763 & 75 \\
11 & 1,11 & 0,333 & 75 \\
12 & 1,11 & 1,193 & 75 \\
13 & 1,11 & 0,763 & 30 \\
14 & 1,11 & 0,763 & 120 \\
15 & 1,11 & 0,763 & 75 \\
16 & 1,11 & 0,763 & 75 \\
17 & 1,11 & 0,763 & 75 \\
\hline
\end{tabular}

A produtividade $y(\%)$ é representada na Equação 1, na qual $m_{c}$ representa a massa $(\mathrm{g})$ de cristais obtida ao final do processo de cristalização e $m_{s}$ a massa (g) de sementes introduzidas no cristalizador no início do processo de cristalização.

$$
y(\%)=\left(\frac{m_{c}}{m_{s}}-1\right) .100
$$

A análise granulométrica dos cristais obtidos foi realizada em um vibrador de peneiras granulométrico (Retsch, modelo AS 200), utilizando peneiras da marca Bertel, com as seguintes aberturas: Tyler 12 $(1,41 \mathrm{~mm})$, Tyler $14(1,19 \mathrm{~mm})$, Tyler 16 $(1,00 \mathrm{~mm})$, Tyler $20(0,841 \mathrm{~mm})$, Tyler 24 $(0,707 \mathrm{~mm})$ e Tyler $28(0,595 \mathrm{~mm})$. A 
operação de peneiramento teve duração de 30 min com amplitude de vibração de $1,5 \mathrm{~mm}$.

O diâmetro médio de Sauter $\left(\bar{D}_{s t}\right)$ foi calculado usando a Equação 2, esta resposta relaciona a fração mássica de sementes retidas $\left(\Delta x_{i}\right)$ com o diâmetro médio da porção retida $\left(D_{i}\right)$ em mm.

$$
\bar{D}_{s t}=\frac{1}{\sum_{i=1}^{p} \frac{\Delta x_{i}}{D_{i}}}
$$

O diâmetro médio do cristal retido em uma determinada peneira $\left(D_{i}\right)$ foi calculado usando a Equação 3, em que $D_{+}$representa o diâmetro de peneira imediatamente superior $(\mathrm{mm})$ e $D$ - o diâmetro $(\mathrm{mm})$ de peneira onde os cristais foram retidos.

$$
D_{i}=\frac{D_{+}+D_{-}}{2}
$$

Das variáveis observadas, a intensidade de vibração pôde ser mensurada através do adimensional de vibração $\Gamma$, calculado através da Equação 4, em que $A$ e $\omega$ são respectivamente, a amplitude de vibração $(\mathrm{m})$ e a frequência angular de vibração $\left(\mathrm{s}^{-1}\right)$ do agente excitador externo que promove a vibração no leito, e $g$ representa a gravidade.

$$
\Gamma=\frac{A \omega^{2}}{g}
$$

O cálculo da supersaturação foi realizado através da Equação 5, na qual $S$ representa o adimensional de supersaturação, $C$ a concentração do sistema na temperatura de operação e $C^{*}$ a concentração de saturação na temperatura do sistema, ambas as concentrações em $\mathrm{kg} / \mathrm{kg}$ de água. Jenkins (1966) afirma que a região considerada metaestável para a cristalização da sacarose está no intervalo de coeficiente de supersaturação $(S)$ de 1,00 e 1,20.
$S=\frac{C}{C^{*}}$

\subsection{Procedimento Experimental do PCC}

Sacarose comercial $\left(\right.$ Caeté $\left.^{\circledR}\right)$ foi utilizada na preparação de soluções de sacarose, em concentração referente ao PCC. Os dados de solubilidade de sacarose em água foram retirados de Oetterer (2006).

Ao término do preparo da solução, uma amostra de $280 \mathrm{~mL}$ foi transferida para o cristalizador batelada, este previamente montado e ajustado para o experimento. Após a transferência da solução para o cristalizador, o aparelho foi vedado e esperou-se até a estabilização da temperatura. A temperatura de operação do processo de cristalização foi de $70^{\circ} \mathrm{C}$, valor mantido constante durante todo o processo.

Com a estabilização da temperatura do meio, $12 \mathrm{~g}$ de cristais de sacarose foram adicionadas à solução no interior do cristalizador. Estes atuaram como sementes no processo de cristalização e foram obtidos por meio do peneiramento do produto comercial, o tamanho das sementes utilizado foi o produto retido na peneira Tyler 20 $(0,841 \mathrm{~mm})$.

Decorrido o tempo de operação, os cristais foram separados da solução usando uma peneira Tyler $24(0,707 \mathrm{~mm})$, e lavados com solução alcoólica nas concentrações de $75 \% \quad\left(1^{\mathrm{a}}\right.$ lavagem $)$ e $96 \% \quad\left(2^{\mathrm{a}}\right.$ lavagem $)$, conforme Pereira (1997). Por fim, o processo de secagem foi realizado em uma estufa por um período de $48 \mathrm{~h}$ a $60^{\circ} \mathrm{C}$.

\subsection{Procedimento Experimental da Cinética de Cristalização}

Após o tratamento de dados dos resultados obtidos experimentalmente pelo PCC, foi determinada a condição otimizada para região de estudo considerada, nesta foi realizada o estudo cinético de crescimento cristalino, mediante o seguinte procedimento: 
Dividiu-se o tempo ótimo de operação em seis intervalos de 14 min, este procedimento foi adotado com o intuito de determinar-se o incremento de dimensão linear $\Delta L_{c f}$ para cada tempo amostral. Nos ensaios, realizou-se o processo de cristalização na condição de resposta máxima, e, atingindo-se o tempo de cada amostra, o processo foi interrompido e os cristais separados da solução.

No desenvolvimento de distribuição granulométrica, há a existência de um único valor de incremento de dimensão linear $\Delta L_{c}$, para uma massa unitária média de cristais $\bar{m}_{c}$, conforme Equação 6, na qual $L_{\mathrm{s}}$ representa o valor do comprimento linear da semente de sacarose, $M_{\mathrm{s}}$ a massa média de uma semente.

$\bar{m}_{c}=\left(1+\frac{\Delta L_{c f}}{L_{s}}\right)^{3} M_{s}$

O valor de $L_{\mathrm{s}}$ foi obtido através do valor médio do comprimento de 50 sementes, medidas com paquímetro digital (King Tools, resolução $0,01 \mathrm{~mm}$ ). A massa média de uma semente, $M_{\mathrm{s}}$, foi determinada através da pesagem de 555 sementes de sacarose em uma balança analítica (Gehaka, modelo BG200, resolução $0,001 \mathrm{~g}$ ) obteve-se o valor médio de $3,67 \times 10^{-6} \mathrm{~kg}$.

A massa unitária média de cristais em uma peneira $\left(\bar{m}_{c}\right)$ foi determinada em todas as faixas granulométricas em cada tempo amostral através da Equação 7, em que $x_{c, t}$ representa a fração mássica retida em cada peneira em uma amostragem, $N_{c}$ o número de cristais e $M_{c}$ a massa total de cristais obtida em cada amostragem.

$$
\bar{m}_{c}=\frac{x_{c, t} M_{c}}{N_{c}}
$$

O cálculo do número de cristais foi realizado usando a Equação 8, na qual $M_{\mathrm{c}}$ representa a massa média de um cristal de sacarose durante o processo de cristalização retido em uma peneira.

$$
N_{c}=\frac{M_{c}}{\bar{m}_{c r}}
$$

O valor de $\bar{m}_{c r}$ foi determinado através da pesagem de 555 cristais de sacarose em 11 quantidades distintas de material, as peneiras utilizadas no estudo de crescimento cristalino foram de Tyler $12(1,41 \mathrm{~mm})$ a Tyler 28 $(0,595 \mathrm{~mm})$, as mesmas utilizadas na obtenção das sementes introduzidas no cristalizador.

\section{RESULTADOS E DISCUSSÃO}

\subsection{Resultados do Planejamento de Experimento}

Os resultados experimentais do Planejamento de Experimentos para produtividade e tamanho final dos cristais de sacarose, são apresentados respectivamente nas Figuras 3 e 4.

Figura 3 - Produtividade alcançada nos experimentos de acordo com o PCC

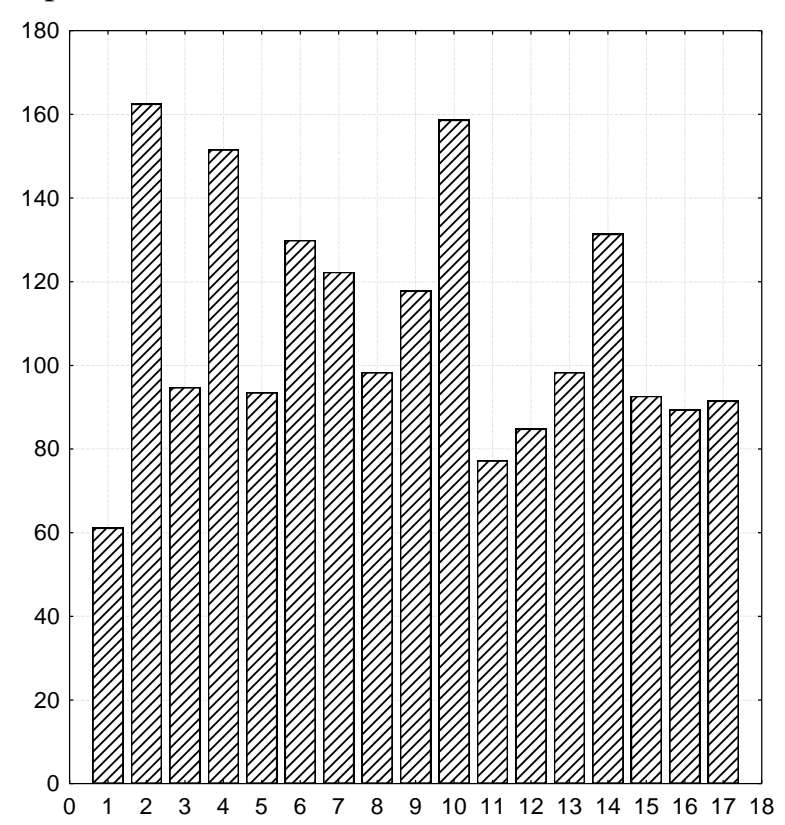


Figura 4 - Diâmetro médio de Sauter obtido nos experimentos de acordo com o PCC.

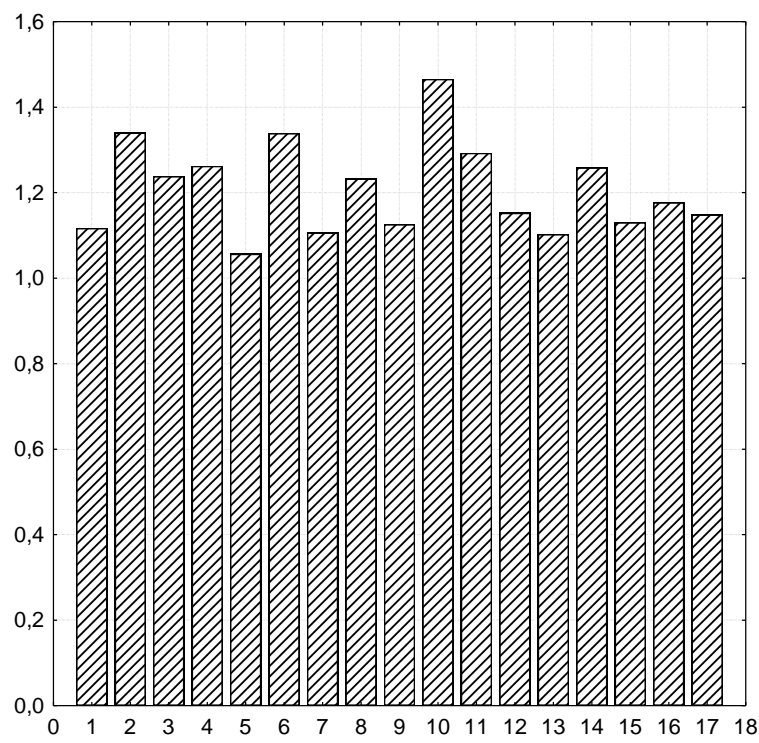

Nas Figuras 3 e 4 observa-se que os resultados nos experimentos 15, 16 e 17 foram semelhantes, demonstrado a reprodutibilidade dos dados em razão destes serem os pontos centrais, isto é, nas mesmas condições operacionais. Em ambas as respostas, obtiveram-se resultados significativos no experimento 10 . Nesta configuração, trabalhou-se com nível máximo de supersaturação e valores intermediários para intensidade de vibração e tempo.

Mediante a análise canônica dos dados obtidos e com auxílio do software Statistica, obteve-se a condição operacional otimizada para ambas as respostas, neste cálculo, foi considerado como "desejabilidade" 1 a resposta máxima para produtividade e diâmetro de Sauter, valores intermediários e mínimos tiveram peso zero. Nesta configuração, de acordo com o modelo obtido, foi necessário elevar a nível máximo a supersaturação, fixar a intensidade de vibração para $308 \mathrm{rpm}$ e tempo de operação de 82 min.

Na Figura 5 observa-se a superfície de resposta para a produtividade, que considera as variáveis supersaturação e tempo de operação, observa-se que a produtividade de cristais é maximizada para altos níveis de supersaturação e menores tempos de cristalização.

Figura 5 - Superfície de resposta das variáveis supersaturação e tempo na resposta produtividade.

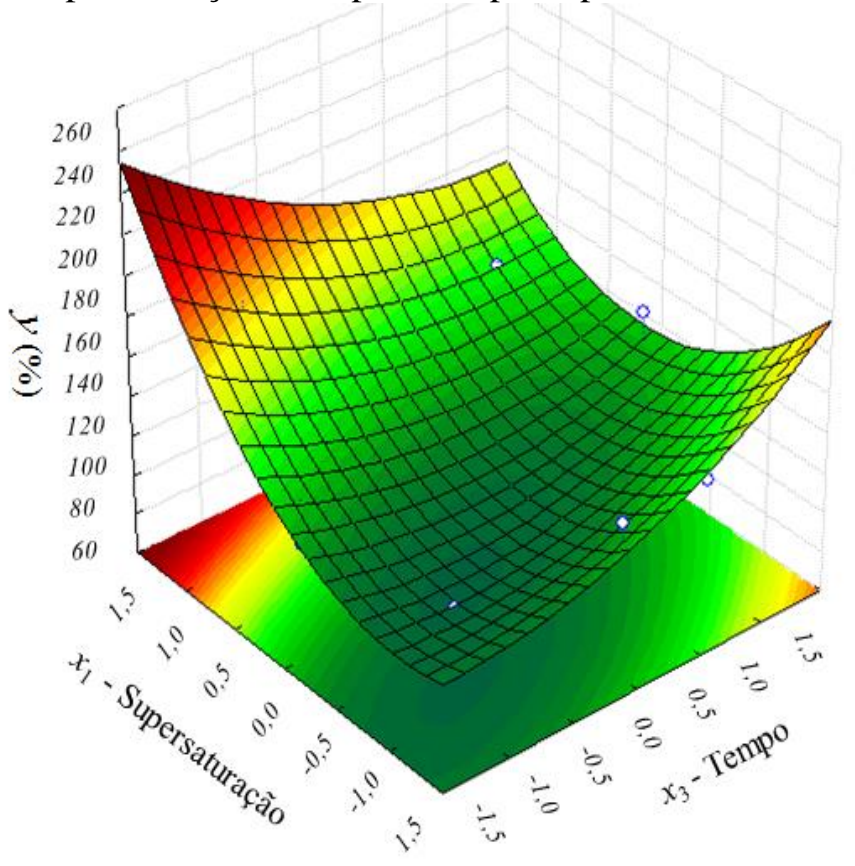

\subsection{Resultados da Análise Cinética}

A análise de crescimento cristalino foi realizada com experimentos na condição otimizada, estes tiveram seus tempos de operação distintos, com diferença de $14 \mathrm{~min}$ em cada teste até atingir-se o tempo final de 82 min. No primeiro experimento, as condições de supersaturação e vibração do meio foram as mesmas da condição na resposta máxima, ao atingir o tempo de 14 min de operação o processo foi interrompido, (0,595 mm) e lavados com solução alcóolica, estes cristais foram definidos como amostras de tempo 14.

Procedeu-se da mesma forma para todos os seis tempos distintos de operação, ao fim, pesando-se os cristais obtidos em cada peneira para cada tempo de cristalização, obteve-se a fração mássica $\left(x_{c, t}\right)$ dos cristais em cada peneira. Via técnica de 
quarteamento, foram selecionados $10 \mathrm{~g}$ de cada amostra, com isso obteve-se a massa de cristais $\left(M_{\mathrm{c}}\right)$ existente em cada peneira. Obtida a massa de cristais, dividiu-se os valores pela massa média de um cristal em cada peneira e obteve-se o número de cristais retido em cada peneira para seis intervalos. Por fim, calculou-se a massa unitária média e o incremento de dimensão linear para peneira, estes últimos valores em função do tempo de cristalização são apresentados na Figura 6.

Figura 6 - Incremento de dimensão linear dos cristais em função do tempo do processo.

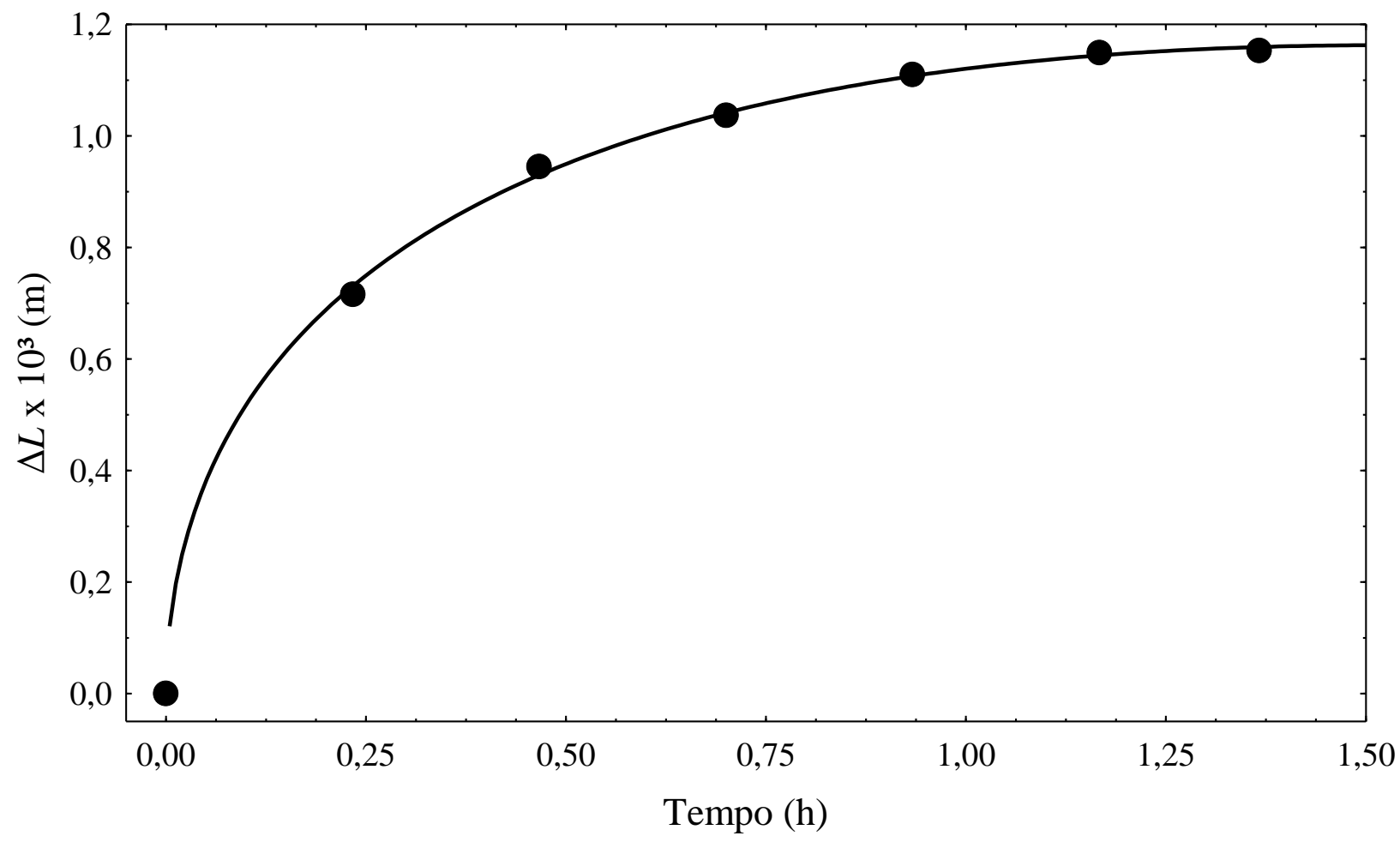

A curva de ajuste dos pontos foi obtida através do software Tablecurve $2 D$, esta é proveniente da curva de ajuste do polinômio na Equação 9, na qual $b$ e $c$ são parâmetros a ajustar. Neste ajuste, o coeficiente de correlação quadrático $\left(\mathrm{R}^{2}\right)$ obtido foi de 0,9956. A Equação 10 apresenta o incremento de dimensão linear com o tempo.

$$
y=b x+c x^{0,5}
$$

$$
\Delta L_{c t}=-0,776 t+1,897 t^{0,5}
$$

A taxa de crescimento linear, $G$, pôde ser obtida através da variação do incremento de dimensão pelo tempo. Diferenciando a equação genérica do ajuste pelo tempo, obteve-se a taxa de crescimento linear, apresentada na Equação 11.

$$
G=\frac{d L_{c t}}{d t}=\frac{d\left(\Delta L_{c t}\right)}{d t}=-0,776+\frac{0,948}{\sqrt{t}}
$$

Os valores da taxa de crescimento em função do tempo de cristalização são apresentados na Figura 7. Observa-se o rápido decaimento da taxa para os primeiros $20 \mathrm{~min}$ de operação, destaca-se que a partir do quinto tempo de amostragem (70 $\mathrm{min})$ não há variação significativa na taxa, fazendo com que o incremento de dimensão linear no cristal tenda a zero. 
Figura 7 - Taxa de crescimento linear cristalino em função do tempo de cristalização.

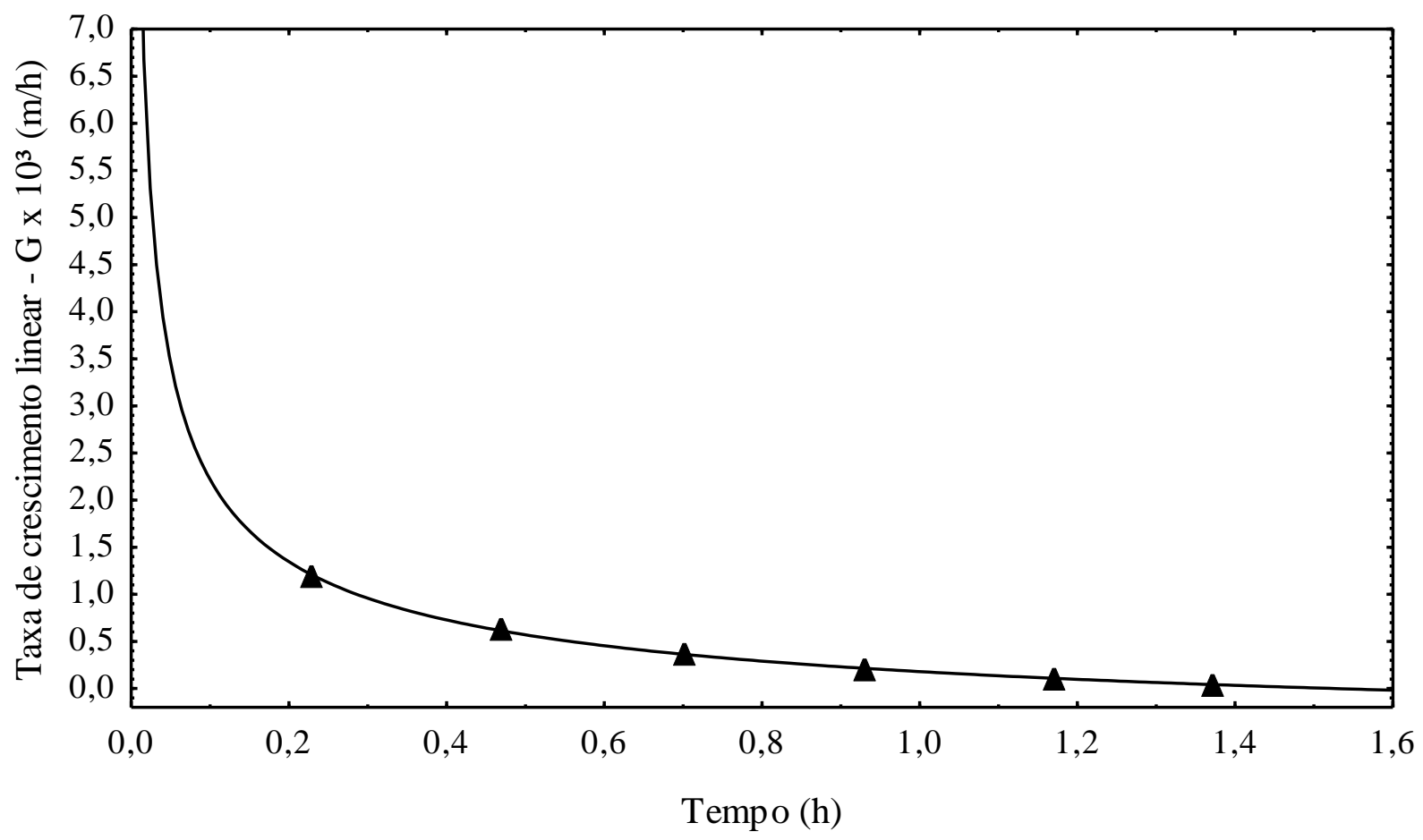

\section{CONCLUSÃO}

Constatou-se que somente a baixa intensidade de vibração favoreceu o aumento da produtividade e do diâmetro médio do cristal para um alto índice de supersaturação. A baixa intensidade de vibração favoreceu a transferência de massa para a superfície cristalina mediante a redução da camada limite em torno do cristal. Para elevada intensidade de vibração em alto índice de supersaturação foi observado queda para ambas as respostas, tal constatação sugere que a alta vibração favoreceu o choque entre os cristais ocasionando quebra, diminuindo consequentemente o tamanho final dos mesmos.

A condição otimizada de operação estabeleceu valor máximo para supersaturação, valor baixo para a intensidade de vibração e intermediário para o tempo, isto é, $S=1,20, \Gamma=0,514$ (308 rpm) e $t=82 \mathrm{~min}$.

A taxa de crescimento linear obtida para esta configuração, $G, \quad$ decresceu significativamente nos primeiros minutos de operação, tal parâmetro não possuiu variação significativa ao final do processo, tendo seu valor tendendo a um valor próximo a zero.

Mediante o tempo de cristalização proposto ser semelhante ao utilizado industrialmente, e também devido ao tamanho médio final dos cristais, a taxa de crescimento demonstrou-se alta, proporcionando um produto com granulometria semelhante ao produto comercial.

\section{NOMENCLATURA}

\begin{tabular}{|l|l|l|}
\hline$A$ & Amplitude de vibração & {$[\mathrm{m}]$} \\
\hline
\end{tabular}




\begin{tabular}{|c|c|c|}
\hline$C$ & $\begin{array}{l}\text { Concentração do } \\
\text { sistema na temperatura } \\
\text { de operação }\end{array}$ & $\begin{array}{r}{[\mathrm{kg} / \mathrm{kg} \text { de }} \\
\text { água] }\end{array}$ \\
\hline$C^{*}$ & $\begin{array}{l}\text { Concentração de } \\
\text { saturação na } \\
\text { temperatura do sistema }\end{array}$ & $\begin{array}{r}{[\mathrm{kg} / \mathrm{kg} \text { de }} \\
\text { água }]\end{array}$ \\
\hline$D_{\mathrm{i}}$ & $\begin{array}{l}\text { Diâmetro médio da } \\
\text { porção retida }\end{array}$ & {$[\mathrm{mm}]$} \\
\hline$g$ & $\begin{array}{l}\text { Aceleração da } \\
\text { gravidade }\end{array}$ & {$\left[\mathrm{m} / \mathrm{s}^{2}\right]$} \\
\hline$G$ & Taxa de crescimento & {$\left[\mathrm{m} \cdot \mathrm{min}^{-1}\right]$} \\
\hline$L_{s}$ & $\begin{array}{l}\text { Dimensão linear de } \\
\text { referência da semente }\end{array}$ & [m] \\
\hline$m_{c}$ & Massa de cristais & {$[\mathrm{g}]$} \\
\hline$M_{\mathrm{c}}$ & $\begin{array}{l}\text { Massa total de cristais } \\
\text { obtidos a cada } \\
\text { amostragem }\end{array}$ & {$[\mathrm{kg}]$} \\
\hline$m_{\mathrm{s}}$ & $\begin{array}{l}\text { Massa de sementes no } \\
\text { PCC }\end{array}$ & {$[\mathrm{g}]$} \\
\hline$M_{\mathrm{s}}$ & $\begin{array}{l}\text { Massa média de uma } \\
\text { semente }\end{array}$ & {$[\mathrm{g}]$} \\
\hline$N_{c}$ & $\begin{array}{l}\text { Número de cristais } \\
\text { presentes da solução }\end{array}$ & {$[-]$} \\
\hline PCC & $\begin{array}{l}\text { Planejamento } \\
\text { Composto Central }\end{array}$ & \\
\hline$S$ & $\begin{array}{l}\text { Coeficiente de } \\
\text { supersaturação }\end{array}$ & {$[-]$} \\
\hline$X c, t$ & $\begin{array}{l}\text { Fração mássica de } \\
\text { cristais retida em cada } \\
\text { peneira }\end{array}$ & {$[-]$} \\
\hline$x_{i}$ & $\begin{array}{l}\text { Variável codificada } \\
\text { analisada }\end{array}$ & {$[-]$} \\
\hline$y$ & $\begin{array}{l}\text { Produtividade de } \\
\text { cristais }\end{array}$ & {$[\%]$} \\
\hline$\Delta L_{\mathrm{c}}$ & $\begin{array}{l}\text { Incremento de } \\
\text { dimensão linear }\end{array}$ & {$\left[\mathrm{m} .10^{3}\right]$} \\
\hline$\Delta x_{\mathrm{i}}$ & Fração mássica retida & \\
\hline$v$ & $\begin{array}{l}\text { velocidade do } \\
\text { excêntrico }\end{array}$ & {$[\mathrm{rpm}]$} \\
\hline$\Gamma$ & $\begin{array}{l}\text { adimensional de } \\
\text { vibração }\end{array}$ & {$[-]$} \\
\hline$\omega$ & frequência de vibração & {$\left[\mathrm{s}^{-1}\right]$} \\
\hline
\end{tabular}

\section{REFERÊNCIAS}

ANDRADE, S. J.; CRISTALE, J.; SILVA, F. S.; ZOCOLO, G. J.; MARCHI, M. R. R. Contribution of sugar-cane harvesting season to atmospheric contamination by polycyclic aromatic hydrocarbons (PAHs) in Araraquara city, Southeast Brazil. Atmospheric Environment, v.44, p.2913-2919, 2010.

CAMILO, O. V.; REYNOSO, B. E.; RODRUIGUES, E.; RAMIREZ, J. A. Characterization of cane sugar crystallization using image fractal analysis. Journal of Food Engineering, v.110, p.77-84, 2010.

Chen, J. C. P.; Chou, C. C. Cane Sugar Handbook: A Manual for Cane Sugar Manufacturer and Their Chemists, New York: John Wiley \& Sons, 1993.

FAO. Food and Agriculture Organization of the United Nations. Food Outlook Biannual report on global food markets, Roma, Itália, 2014. Disponível em: <http://www.fao.org/3/a-i4136e.pdf >. Acesso em 25 de mar. 2015.

JENKINS, G. H., Introduction to cane sugar technology. Elsevier Science Publishing, v.1, p.265-408, 1966.

JONES, A. G. Crystallization process systems. Oxford: Butterworth-Heinemann, 2002.

MALAGONI, R. A. Cristalização de ácido cítrico em leito vibrado. 2010. 297p. Tese (Doutorado em Engenharia Química), Universidade Federal de Uberlândia, Uberlândia, 2010.

MULliN, J. W. Crystallization. Oxford: Butterworth - Heinemann, 2001. 
MYERSON, A. S. Handbook of Industrial Crystallization. Boston: ButterworthHeinemann, 2002.

NAGY, K. Z.; FEVOTTE, G.; KRAMER, H., SIMON, L. L. Recent advances in the monitoring, modeling and control of crystallization system. Chemical Engineering Research and Design, v.91, p.1903-1922, 2013.

OETTERER, M., SARMENTO, R. d'D., M. A., SPOTO, M. H. F. Fundamentos de Ciência e Tecnologia de Alimentos, Barueri: Manole, 2006.

OUIAZZANE, S.; MESSNAOUI, B.; ABDERAFI, S.; WOUTERS, J.; BOUNAHMIDI, T. Estimation of sucrose crystallization kinetics from batch crystallizer data. Journal of Crystal Growth, v.310, p.798-803, 2007.

PEREIRA, A. G. Cristalização de sacarose em leito vibro - jorrado. 1997. 144p. Dissertação (Mestrado em Engenharia Química) - Universidade Federal de Uberlândia, Uberlândia, 1997.

QUINTAS, M.; BRANDÃO, T. R. S.; SILVA, C. L. M.; CUNHA, R. L. Rheology of supersaturated sucrose solutions. Journal of Food Engineering, v.77, p.844-852, 2005.

QUINTINO, D. B. Otimização operacional do processo de cristalização da sacarose em leito vibrado. 2014. 96p. Dissertação (Mestrado em Engenharia Química) Universidade Federal de Uberlândia, Uberlândia, 2014.

TEIXEIRA, G. A.; VIEIRA, W. F.; FINZER, J. R. D.; MALAGONI, R. A. Citric acid crystallization process in dense phase using vibrated bed. Journal of Food Engineering, v.111, p.458-465, 2012.
ULRICH, J.; JONES, M. J. Industrial crystallization developments in research and technology. Chemical Engineering Research and Design, v.82, p.1567-1570, 2004.

\section{AGRADECIMENTOS}

Este trabalho foi financeiramente apoaiado pela CAPES. 